(2) 
Heinz Politzer

\title{
DAS SCHWEIGEN DER
}

\section{SIRENEN}

\author{
STUDIEN ZUR \\ DEUTSCHEN UND ÖSTERREICHISCHEN \\ LITERATUR
}

J. B. METZLERSCHE

VERLAGSBUCHHANDLUNG

STUTTGART 
ISBN 978-3-476-99754-8

ISBN 978-3-476-99753-1 (eBook)

DOI 10.1007/978-3-476-99753-1

(C)

Springer-Verlag GmbH Deutschland 1968

Ursprünglich erschienen bei J. B. Metzlersche Verlagsbuchhandlung und Carl Ernst Poeschel Verlag GmbH in Stuttgart 1968 
Vorwort . . . . . . . . . . . . . . . . . 7

Das Schweigen der Sirenen . . . . . . . . . . . . 13

Wer hat Angst vor dem bösen Franz? Kafka erscheint im

Osten . . . . . . . . . . . . . . . . . . . . . 42

Zeit, Wirklichkeit und Musik im Werk Heimito von Doderers. Eine Gedenkrede . . . . . . . . . . . . . . 70

Menschwerdung eines Schriftstellers. Zu Heimito von Doderers Divertimento »Die Posaunen von Jericho« . . . 79

Hofmannsthals Vorspiel zu Brecht . . . . . . . . . . 100

Diagnose und Dichtung. Zum Werk Arthur Schnitzlers . 110

Der arme Hofrat. Franz Grillparzer in seinen Tagebüchern 142

Alt-Wiener Theaterlieder . . . . . . . . . . . . . . 160

Zauberspiegel und Seelenkranker. Ferdinand Raimunds

»Der Alpenkönig und der Menschenfeind« . . . . . 185

Um einen Heine von innen bittend . . . . . . . . . . 206

Szene und Tribunal. Schillers Theater der Grausamkeit 234

Vom Baum der Erkenntnis und der Sünde derWissenschaft.

Über Goethes »Faust« . . . . . . . . . . . . . . 254

„Kein Mensch ist ein Eiland“. Zu Goethes »Iphigenie« . 285

Gretchen im »Urfaust $«$. . . . . . . . . . . . . . 312

Lessings Parabel von den drei Ringen . . . . . . . . 339

Das Handwerk der Interpretation. Ein Vortrag . . . . . 373

Anmerkungen . . . . . . . . . . . . . . . . . . 399

Personenregister . . . . . . . . . . . . 430 


\section{VORWORT}

Die Entstehungsgeschichte der hier vorliegenden Arbeiten umspannt eine beträchtliche Reihe von Jahren. Partien des »Heine«-Aufsatzes gehen auf Versuche zurück, die ich in den frühen Vierzigerjahren in Jerusalem im Zusammenhang mit einer Literaturgeschichte der deutsch-jüdischen Symbiose unternommen habe. Die meisten anderen Aufsätze sind in Amerika entstanden, mehr oder minder als Resultate meiner Tätigkeit als Lehrer der deutschen Literatur am Bryn Mawr College (Pennsylvania), am Oberlin College (Ohio) und an der Universität von Kalifornien in Berkeley. Die Gedenkrede auf Heimito von Doderer habe ich erst im Dezember 1967 gehalten.

Es ist also ein ganzes Stück Leben mit seinen Wandlungen und Widersprüchen, aus dem diese Studien erwachsen sind. Gemeinsam ist ihnen die Bindung an die deutsche Sprache und ihre Dichtung, eine Bindung aus der Ferne. Absence makes the heart grow fonder. Ich bin nicht sicher, ob sich der populäre Satz, dem zufolge Abwesenheit das Herz zärtlicher schlagen läßt, auch den überpersönlichen Gebilden des Geistes gegenüber unbedingt aufrechterhalten läßt. Wohl aber habe ich gemerkt, daß Distanz das Auge dazu zwingt, seinen Blick zu schärfen. Distanz ist es gewesen, was meine Studien erleichtert, wenn nicht gar ermöglicht hat.

Gemeinsam ist diesen Essays ferner die Frage, die sie offen oder andeutungsweise stellen: sie beschäftigen sich letzten Endes mit der Ergründbarkeit des dichterischen Wortes durch das deutende. Was das dichterische und was das kritische Wort zu sagen hat, glaube ich aus eigener Erfahrung zu wissen. Der Titelaufsatz des Bandes versucht, durch die Verfolgung eines literarischen Motivs und seiner Verzweigungen den Weg nachzuzeichnen, der die 
Dichter vom gestaltenden Wort $\mathrm{zu}$ dem im Wort gestalteten Schweigen geführt hat. Der Interpretation des Schweigens, dieses Vorrechts aller großen Dichtung, und der durch diese Interpretation akzentuierten Frage nach der Möglichkeit, das dichterische Wort bis zum Grunde zu erhellen, kommt, wie ich zu zeigen hoffe, grundsätzliche Bedeutung zu in einer Zeit, in der die Dichtung, und bei weitem nicht die deutsche Dichtung allein, an ihre „Atemwende" gelangt ist.

Der Versuch, eine solche Frage 'theoretisch zu beantworten, bedürfte eine jener systematischen Abhandlungen, wie sie seit längerem schon von Linguisten, Phänomenologen und Ontologen verfaßt worden sind. Ich denke hier unter anderem an die Untersuchungen meines Freundes und Kollegen Andrew O.Jászi ${ }^{1}$, der mir für den Aufgabenkreis und die Methode ontologischer Deutung von Dichtung die Augen geöffnet hat. Aber die Absicht meiner Studien ist nicht ästhetischer oder poetologischer Art; sie bieten Beispiele aus der Praxis des Deutens, individuelle Interpretationen individueller Texte und Gestalten oder, wie der Aufsatz »Wer hat Angst vor dem bösen Franz?«, einer spezifischen Wirkungsgeschichte. Der Essay über die »Alt-Wiener Theaterlieder « bildet nur insofern eine Ausnahme, als er die Interpretation eines Genres, wenn auch eines ganz besonderen, zum Gegenstand hat.

Wie ich bei diesen Deutungen vorgegangen bin, habe ich in dem Schlußstück der Sammlung, dem »Handwerk der Interpretation «, dargestellt, einem Vortrag, den ich in Amerika und dann auch in Deutschland gehalten habe. In Zustimmung und Abgrenzung ist er bekenntnishafter Natur, wie Vorträge dies sein dürfen und sollen. Dabei hat es mich gefreut zu beobachten, daß zumindest eine seiner Forderungen, die nach „philologischem Takt", von vielen meiner Hörer hüben und drüben immer noch oder schon wieder geteilt wird.

Etwa die Hälfte dieser Aufsätze ist Themen aus der österreichischen Literatur gewidmet. Trotz des Hofmannsthalschen Schemas »Preuße und Österreicher ${ }^{2}{ }^{2}$ trotz Josef Nadlers »Literaturgeschichte Österreichs $\ll^{3}$, und der scharfsinnig-beredten Glossen 
Gerhart Baumanns zur ,österreichischen Geistesverfassung“4 um nur die mir nächstliegenden Erläuterungen zu nennen vermag ich nicht mit Bestimmtheit zu sagen, was die Wesensart des Landes ist, aus dem ich komme, und worin die "Geistesverfassung" einer Literatur besteht, deren Darstellung ich einen so breiten Raum zumesse. Dennoch gibt es diese Art, diese Verfassung; sie sind ein Teil von mir und so ein Teil dieser Studien. Das Österreichische ist mehr als Tradition und Landschaft, mehr als Erbe und Tonfall; die Neigung zur ,Flucht vor der Größe“ 5, zum ,Selbstgericht" " , herrscht zwar in seiner Literatur vor, erschöpft jedoch keineswegs ihre Eigenart. Niemals bin ich mir dieser Tatsache mehr bewußt worden, als in jener Märznacht des Jahres 1938, in der dieses sonderbare Wesen mir meine Zugehörigkeit zu ihm aufkündigte. Die Kündigung erwies erst, daß Ursprünge unverlierbar und echte Bindungen nicht einseitig aufzuheben sind. Verfolge ich die Chronologie meiner Studien (der Leser findet sie im Anmerkungsteil dieses Bandes verzeichnet), dann will es mir beinahe scheinen, als träte dieses Österreichische umso deutlicher hervor, je weiter die Jahre der Entfernung fortschritten.

Anläßlich seines »König Ottokar « trug Grillparzer 1825 in sein Tagebuch ein: „Nur ein Thor könnte glauben, daß dem Dichter hier die Verknüpfung von Ursache und Wirkung erlassen wäre. Aber wie in der Natur sich höchst selten Ursache und Wirkung wechselseitig ganz decken, so ist, in der Behandlung eine gewiße Inkongruenz Beider durchblicken zu lassen, villeicht die höchste Aufgabe, die ein Dichter sich stellen kann. Allerdings eine höchst gefährliche Klippe! Die Unverständlichkeit, der Unsinn lauern geschäftig auf jeden Fehltritt, und nur die Anschauung kann retten, indeß der Begriff rein nutzlos wird, und zurückbleibt ... Es müssen ferner die gewagten (scheinbaren) Inkonsequenzen, eigentliche Inkonsequenzen der Natur seyn, und der Zuschauer muß das Gesetz der Kaussalität fühlen, wenn er es auch nicht nachweisen kann. " " Die Forderung nach dem Konkreten und dem Sinn für das Komplexe, die Grillparzer hier für sein historisches 
Drama erhebt, scheinen mir verbindliche Züge einer Literatur, die schon in diesem Dichter nach dem Wesen der Sprache gefragt (»Weh dem, der lügt!«), die Botschaft des Schweigens gehört und in wortloser Gebärde vorbildlich ausgesprochen hat.

Was aber für die Literatur gilt, gilt in gleichem Maße für eine Kritik, die dieser Literatur gerecht werden will. Mir scheint die Reflexion des österreichischen Dichters darauf hinzudeuten, daß eine solche Kritik die strengen Gesetze von Dichtung und Deutung anzuerkennen und zu bewahren habe; auch sie steuert stets zwischen „höchst gefährlichen Klippen“. Dennoch steht sie vor der Aufgabe, dem Organisch-Gewachsenen, der Natur des Schöpferischen Rechnung zu tragen, indem sie jene Gesetze weder als a priori gegeben noch als absolut voraussetzt. Durch die ihr innewohnenden ,gewißen Inkongruenzen“ unterscheidet sich Dichtung vom Gedankensystem, wie sich der echte Apfel durch die gewissen Unebenheiten seiner Schale, durch die kaum merkliche Asymmetrie seines Kerns vom wächsernen unterscheidet. (Ist diese Asymmetrie nicht geradezu eine Gewähr für die Existenz des Kerns?) Kritik entwickelt sich aus dem Schaffensvorgang der Dichtung, wie diese sich in einer Deutung weiterentwickelt, die das Ereignis der Schöpfung in seiner Totalität zu erfassen versucht.

Gewiß wird der Kritiker, der sich seinen Sinn für die Inkongruenzen und Inkonsequenzen der Dichtung bewahrt hat, in seiner Interpretation schwerlich zu völliger Eindeutigkeit vordringen. Und vielleicht ist die Bereitschaft, das Vieldeutige und Frag-Würdige, die Aufgeschlossenheit auch als geschlossen tradierter Dichtung wahrzunehmen, ein österreichischer Beitrag zu diesen Studien, der sich selbst dort nicht zur Gänze unterdrücken ließ, wo Texte interpretiert wurden, die der deutschen Dichtung angehören. Jedenfalls finden diese Versuche, Deutung als einen nie enden wollenden Prozeß des Verstehens von Dichtung zu betreiben, in den der Deutende schließlich miteinbezogen wird, obwohl er sich mit allen Kräften und Mitteln seiner Rationalität dagegen gewehrt hat, in der Maxime Grillparzers eine willkommene Unterstützung. 
Diese Erwägungen blieben unvollständig ohne einige Worte des Dankes an

meine amerikanischen Kollegen und Studenten, die mich durch Gespräch und Kritik zu immer neuen Perspektiven meiner Frage und zur Präzisierung meiner Fragestellung angeregt haben;

die John Simon Guggenheim Memorial Foundation, die es mir in den Jahren 1957-1958 und 1966-1967 durch Stipendien ermöglicht hat, einige meiner Aufsätze zu schreiben und andere auszuarbeiten;

die Professoren, Assistenten und Hörer der deutschen und österreichischen Hochschulen, vor allem meine Freunde an der Albert-Ludwigs-Universität in Freiburg im Breisgau, die es mir im Jahre 1966-1967 erlaubt haben, in öffentlichen Vorträgen Wirkung und Grenzen meiner Studien zu erproben

und die J.B. Metzlersche Verlagsbuchhandlung in Stuttgart, namentlich Herrn Winfried Hellmann, der mir mit wahrhaft produktiver Kritik bei der Auswahl und Revision dieser Essays als unermüdlicher Berater und selbstloser Freund zur Seite gestanden ist.

Ebenso danke ich Fräulein Ursula Dzierson in Freiburg, Frau Hedwig Doehring in Berkeley und Fräulein Irmgard Moritz in Stuttgart, die meine Manuskripte und Druckvorlagen mit oft großer Mühe ins Reine gebracht haben. Fräulein Moritz hat außerdem die Freundlichkeit besessen, das Namensregister dieses Bandes herzustellen. Schließlich weiß ich mich auch Frau Ursula Brent und Frau Hendrika Samuels sowie meinem Kollegen Herrn Dr. Hinrich C. Seeba, die mir beim Lesen der Korrekturen geholfen haben, für ihren Beistand zu innigem Dank verpflichtet. 